\title{
Computational prediction of miRNA in Gmelina arborea and their role in human metabolomics
}

\author{
Alka Dubey ${ }^{1,}$, Swinderjeet Singh Kalra ${ }^{2}$, Nandita Trivedi ${ }^{3}$ \\ ${ }^{1}$ Department of Zoology, Bioinformatics infrastructure facility centre (DBT), Ministry of Science and Technology (Govt. of India), D.G \\ (PG) College civil lines Kanpur, INDIA \\ ${ }^{2}$ Department of Chemistry, D.A-V, College Kanpur INDIA \\ ${ }^{3}$ Department of Biotechnology, CSJM University, Kanpur INDIA
}

Email address:

Alkabioinfo964@gmail.com (A. Dubey )

\section{To cite this article:}

Alka Dubey, Swinderjeet Singh Kalra, Nandita Trivedi. Computational Prediction of miRNA in Gmelina Arborea and their Role in Human Metabolomics. American Journal of Bioscience and Bioengineering. Vol. 1, No. 5, 2013, pp. 62-74.

doi: 10.11648/j.bio.20130105.12

\begin{abstract}
Micro RNA are the noncoding class of RNA which plays major role post transcriptional regulation of genes and 19-22 nt in length and endogenous in nature .In this study miRNA Gmelina arborea are predicted along with possible target genes, A total of 44 ESTs are taken from dbEST database and proceed and trimmed through EGassembler. This contig sequence was now used to find out the putative miRNA by performing a local alignment with miRNA of Arabidopsis thaliana retrieved from miRBase. The targets were scanned by hybridizing screened ESTs with the UTRs of human using DIANA tar software. Finally 6 putative miRNAs were found to hybridize with the various targets of signal transduction and apoptosis that play significant role in preventing disease like cancer, blood borne disease, and other urinary infections and various study indicated that is possesses antioxidants, diuretic, anthelmintic activity, cardio protective, anti diabetic, immunomodulatory, antipyretic and analgesic, antimicrobial in the treatment of jaundice, piles bases on their targets
\end{abstract}

Keywords: Gmelina Arborea, miRNA, Dbest, DIANA, Local Alignment

\section{Introduction}

\subsection{About miRNA}

MicroRNA is a family of small non-coding RNAs that regulate gene expression in a sequence-specific manner. miRNAs are a class of post-transcriptional regulators. miRNAs are a family of 19 to 25 small [1] miRNAs function via base-pairing with complementary sequences within mRNA molecules, usually resulting ingene silencing via translational repression or target degradation.[2] in plant miRNA biogenesis in plants differs from animal biogenesis mainly in the steps of nuclear processing and export. Instead of being cleaved by two different enzymes, once inside and once outside the nucleus, both cleavages of the

Plant miRNA is performed by a Dicer homolog, called Dicer-like1 (DL1). DL1 is only expressed in the nucleus of plant cells, which indicates that both reactions take place inside the nucleus. Before plant miRNA:miRNA* duplexes are transported out of the nucleus, its $3^{\prime}$ overhangs are methylated by a RNA methyl transferaseprotein called Hua-Enhancer1 (HEN1). The duplex is then transported out of the nucleus to the cytoplasm by a protein called Hasty (HST), an Exportin 5 homolog, where they disassemble and the mature miRNA is incorporated into the RISC.[3]

\subsection{Gmelina Arborea. a High Medicinal Value Herb}

The root and bark of Gmelina arborea are claimed to be stomachic, galactagogue laxative and anthelmintic; improve appetite, useful in hallucination, piles, abdominal pains, burning sensations, fevers, 'tridosha' and urinary discharge. Leaf paste is applied to relieve headache and juice is used as wash for ulcers.Flowers are sweet, cooling, bitter, acrid and astringent. They are useful in leprosy and blood diseases.In Ayurveda, it has been observed that Gamhar fruit is acrid, sour, bitter, sweet, cooling, diuretic tonic, aphrodisiac, alternative astringent to the bowels, promote growth of hairs, useful in 'vata', thirst, anaemia, leprosy, ulcers and vaginal discharge. The plant is recommended in combination with other drugs for the treatment of snakebite 
and scorpion sting. In snakebite a decoction of the root and bark is given internally. [4]

\section{Materials and Methods}

The EST sequences of Gmelina arborea were retrieved from dbEST (NCBI), for clean the EST via EG assembler (seq cleaner) were use to find out the contig files and the precursors and secondary energy (MFE)was found with the help of RNA fold then we use to find out the putative miRNA from Gmelina arborea via the local alignment with Arabidopsis thaliana this miRNA predicted the target site ,and the target site prediction and UTRs hybridization and chromosomes position analysis with involves gene metabolic activity via the KEGG path way analysis completed with the help of DIANA web server . miRNA genes can be searched by motif searches combining sequence, structure and conservation information. In order to facilitate the investigation into miRNA function, numerous bioinformatics methods were developed in order to allow high throughput prediction of miRNA target genes. Most miRNA target prediction algorithms use similar general principles in the development of their algorithm. Most algorithms search for targets in the 3'-UTR region of mRNAs, where almost miRNA-target interactions occur in vertebrates. Algorithms usually account for the possibility of multiple target sites for more than one miRNA in each mRNA 3'-UTR region, though they differ in the degree in which combinations of miRNA target sites are incorporated into the prediction algorithm. The degree of sequence conservation is another criterion commonly used to filter possible miRNA targets. Most target prediction algorithms identify orthologous 3'-UTR sequences and check whether the miRNA-target interaction is conserved between closely related species. Many algorithms depend on an initial input of a miRNA. Earlier work shows that miRNA regulates gene expression by targeting the 3' untranslated region of specific messenger RNAs for degradation or translational repression. For the prediction of miRNA in Gmelina arborea and the study of its effect on human metabolomics, a series of computational tools was used.[5]

\subsection{Gmelina Arborea EST Dataset}

44 raw ESTs sequences of Gmelina arborea were downloaded from dbEST (Database of Express Sequence Tags). ESTs database has highest numbers of impurities associated with them which has to be removed for further processing. To remove impurities it was processed through SeqClean [6] a vector removing and trimming tool. It removes non redundant sequences and polyA tails. After that 44 ESTs were obtained. Out of these sequences 40 were trashed and 1 sequence was trimmed and then after this ESTs were processed to Repeat Masker for masking the repeated squence. It is a program that screens DNA sequences for interspersed repeats and low complexity quences for interspersed repeats and low complexity DNA sequences. The output of the program is a detailed annotation of the repeats that are present in the query sequence as well as amodified version of the query sequence in which all the annotated repeats have been masked(default: replaced by Ns). Masked file was a input for the TGICL [7] a clustering tool which clustered the sequences in masked file and made contig and ace files. Clustering of ESTs is must for gene prediction and to know about the functional annotations and to understand the important genetic information's with variations such as those which are involved in diseases.

\section{Results}

Computational prediction of miRNA in Gmelina arborea and their role in human metabolomics analysis depends

Upon different computational prediction analysis.

\subsection{Analysis of Retrieved ESTs for Gmelina Arborea}

For this analysis EST retrieves from dbEST via NCBI according raw data $\mathrm{GA}($ Gmelina arborea) carrying a 44 sequences An expressed sequence tag or EST is a short sub-sequence of a cDNA sequence. They may be used to identify gene transcripts, and are instrumental in gene discovery and gene sequence determination. [8]The identification of ESTs has proceeded rapidly, with approximately 74.2 million ESTs now available in public databases.

\subsection{Analysis of Sequence Cleaner}

Because ESTs database has highest numbers of impurities associated with them which has to be removed for further processing. To remove impurities it was processed through Seq Cleaner (EG assembler) then analyze the basic five outputs like sequence cleaning, repeat masking, vector masking, organelle masking, sequence was assembling.

\subsection{Strategy to Predict Mature miRNA and their Targeted Genes}

The miRNAs of Arabidopsis thaliana were compared with the assembled ESTs of Gmelina arborea to identify regions in the human genome where experimentally validated miRNAs of $A$. Thaliana shows its presence in the contigs of Gmelina arborea. Both mature and precursor miRNA matches were checked out in the resultant clustered contigs and singletons. The resultant miRNAs was compared with the other miRNAs through clastal omega searches to generate a consensus for the predictions of novel mature miRNAs basis on conserved regions and alignments between Arabidopsis thaliana miRNAs and Gmelina arborea contigs precursors the putative miRNA given below . 
Table 1: miRNA sequence obtained through contig file and local alignments

\begin{tabular}{|c|l|l|}
\hline Serial numbers & Annotated name of miRNA & miRNA seq in nucleotide \\
\hline $\mathbf{1 .}$ & miRNA1_GA_CONTIG1 & UGGUAGGGCCCUUCUGAA \\
\hline $\mathbf{2}$. & miRNA2_GA_CONTIG1 & GGCACCGCUGCCAGCGGUAU \\
\hline $\mathbf{3}$. & miRNA3_GA_CONTIG1 & AUGCUGUUUUGGUGGUAGGGCCC \\
\hline $\mathbf{4}$. & miRNA4_GA_CONTIG1 & GAAUUGCAGAAUUCAG \\
\hline $\mathbf{5}$. & miRNA5_GA_CONTIG1 & GCAUGCCUGUCCGAGC \\
\hline $\mathbf{6}$. & miRNA6_GA_CONTIG1 & ACUCAAGACUCUUUUGUUCU \\
\hline
\end{tabular}

Table 1 explains : first coulam explains the serial number and second columns explains the annotated name of miRNA where miRNA as micro RNA and GA as Gmelina arborea and CONTIG means are given RNAs Obtained from contig 1 and Colum thirds explains the nucleotide sequence as miRNA

Table: 2. Name of target genes via putative miRNA from Gmelina arborea

\begin{tabular}{|c|c|c|c|c|}
\hline \multicolumn{5}{|c|}{ >miRNA1_GA_CONTIG1 (UGGUAGGGCCCUUCUGAA) } \\
\hline S.No & Names Of Genes & Position On Chromomsome & 3'UTR & Target Sites Involved In Conserving Information \\
\hline \multirow{8}{*}{1.} & \multirow{2}{*}{ SH3PXD2A } & 10: $105351051-105351079$ & $483-511$ & Not Conserved \\
\hline & & 10: $105349750-105349778$ & $1784-1812$ & Not Conserved \\
\hline & (SH3 and PX domain-containing & 10: $105349745-105349773$ & $1789-1817$ & Not Conserved \\
\hline & protein 2A (SH3 multiple domains & 10: $105347560-105347588$ & $3974-4002$ & oryCun1 \\
\hline & protein 1) (Five & 10: $105347307-105347335$ & $4227-4255$ & Not Conserved \\
\hline & \multirow{3}{*}{$\begin{array}{l}\text { domain-containing protein) (Adaptor } \\
\text { protein TKS5). }\end{array}$} & r10: $105346886-105346914$ & $4648-4676$ & canFam2, loxAfr1 \\
\hline & & 10: $105345560-105345588$ & $5974-6002$ & Not Conserved \\
\hline & & 10: $105344507-105344535$ & $7027-7055$ & Not Conserved \\
\hline \multirow{4}{*}{2.} & \multirow{4}{*}{$\begin{array}{l}\text { ZNF275 } \\
\text { (Zinc finger protein 275.) }\end{array}$} & X: 152270243 - 152270271 & $3817-3845$ & monDom4 \\
\hline & & X: $152270400-152270428$ & $3974-4002$ & Not Conserved \\
\hline & & X: 152270484 - 152270512 & $4058-4086$ & Not Conserved \\
\hline & & X: 152271494 - 152271522 & $5068-5096$ & $\mathrm{rn} 4, \mathrm{~mm} 8$, oryCun1, canFam2 \\
\hline \multicolumn{5}{|c|}{ >miRNA2_GG_CONTIG1 (GGCACCGCUGCCAGCGGUAU) } \\
\hline \multirow[b]{3}{*}{1.} & $\begin{array}{l}\text { MAPK1 } \\
\text { Mitogen-activated protein kinase } 1 \text { (EC }\end{array}$ & 22: 20444943 - 20444971 & $3567-3595$ & $\begin{array}{l}\text { oryCun1, bosTau2, canFam2, dasNov1, loxAfr1, echTel1, } \\
\text { galGa12, xenTro1 }\end{array}$ \\
\hline & (Extracellular & r22: 20447232 - 20447260 & $1278-1306$ & mm8, oryCun1, canFam2, xenTro1 \\
\hline & $\begin{array}{l}\text { signal-regulated kinase } 2) \text { (ERK-2) } \\
\text { (Mitogen-activated protein kinase 2) } \\
\left(\begin{array}{lll}\text { MAP kinase } & 2) \\
(\text { MAPK } & 2) \\
(\text { (p42-MAPK) (ERT1). }\end{array}\right.\end{array}$ & $22: 20444382-20444410$ & $4128-4156$ & Not Conserved \\
\hline \multicolumn{5}{|c|}{ >miRNA3_GA_CONTIG1 (AUGCUGUUUUGGUGGUAGGGCCC) } \\
\hline \multirow{5}{*}{1} & \multirow{5}{*}{$\begin{array}{l}\text { DST } \\
\text { (Bullous pemphigoid antigen 1) }\end{array}$} & 6: $56435863-56435891$ & $14487-14515$ & $\begin{array}{l}\text { rn4, mm8, oryCun1, bosTau2, canFam2, dasNov1, } \\
\text { loxAfr1, echTel1, galGal2, xenTro1, tetNig1 }\end{array}$ \\
\hline & & $\begin{array}{l}6: 56509694 ; 56524941 \\
56509697 ; 56524966\end{array}$ & $8200-8228$ & oryCun1, canFam2, loxAfr1 \\
\hline & & 6: $56571310-56571338$ & $3455-3483$ & canFam2, galGal2 \\
\hline & & 6: $56505225-56505253$ & $8570-8598$ & canFam2, loxAfr1, echTel1 \\
\hline & & 6: $56481326-56481354$ & $10774-10802$ & oryCun1, galGal2 \\
\hline
\end{tabular}




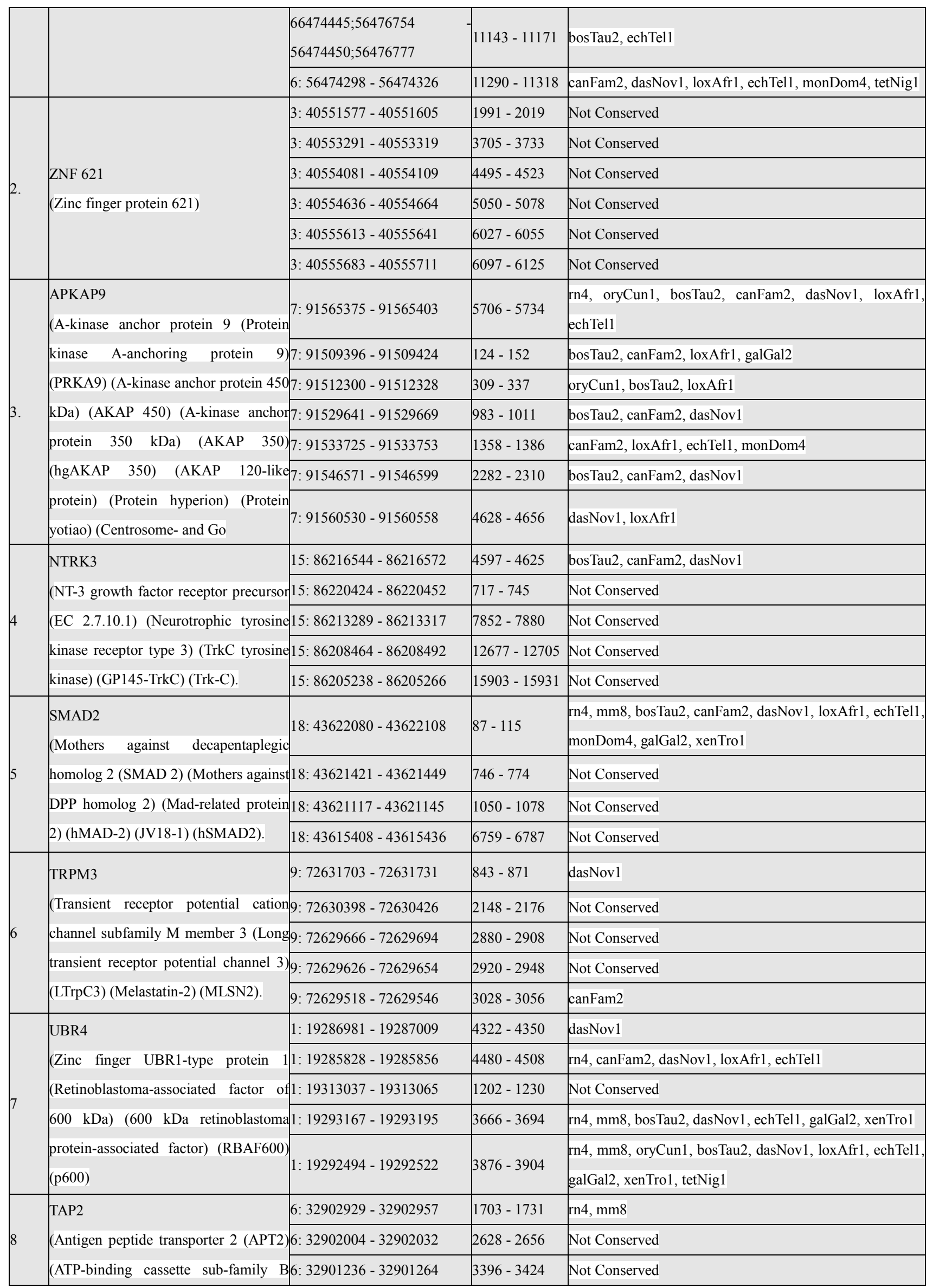




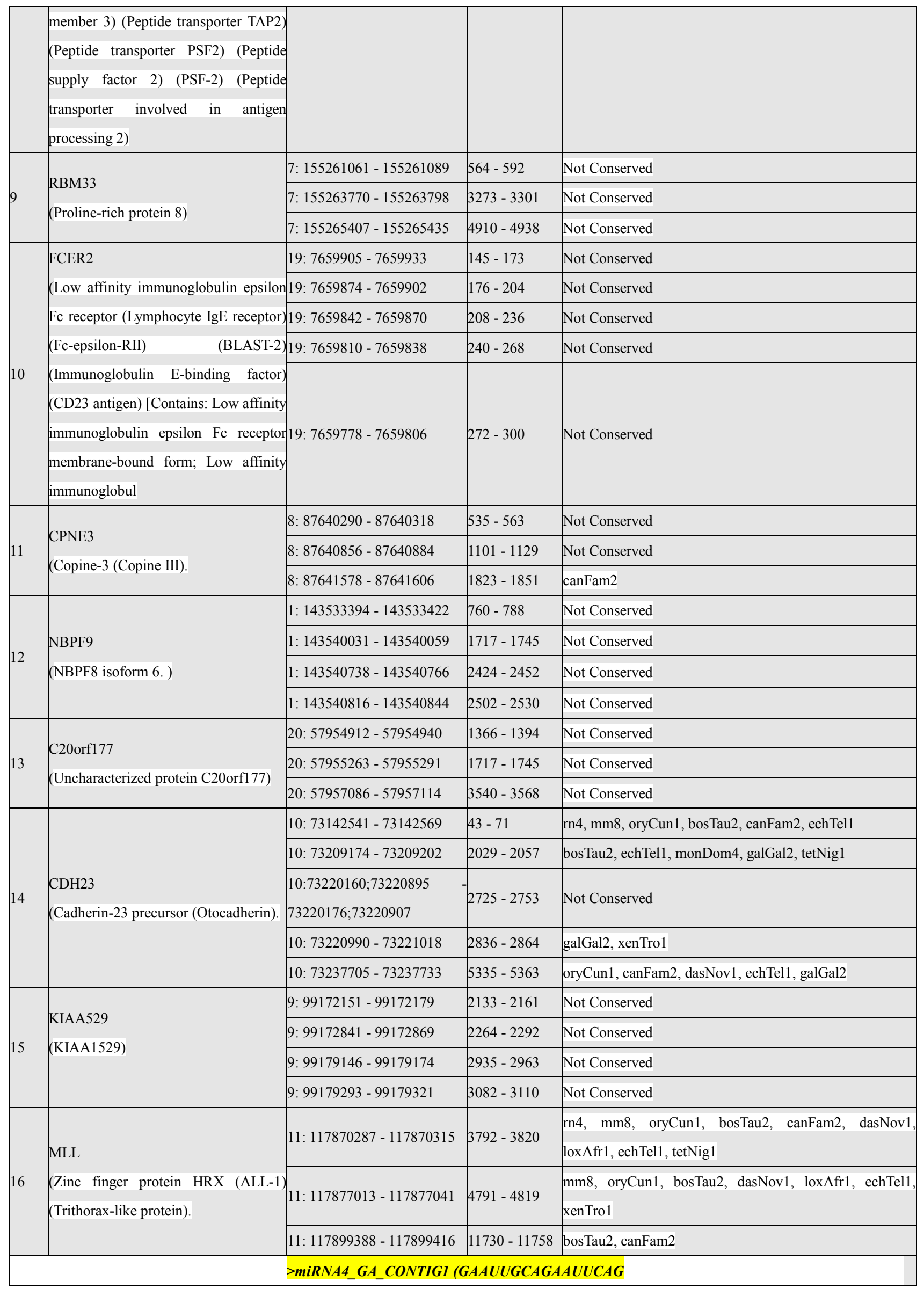




\begin{tabular}{|c|c|c|c|c|}
\hline \multirow{4}{*}{1} & \multirow{4}{*}{$\begin{array}{l}\text { ESSRG } \\
\text { (Estrogen-related receptor gamma } \\
\text { (Estrogen receptor-related protein 3) } \\
\text { (ERR gamma-2). }\end{array}$} & 1: $214745325-214745353$ & $1550-1578$ & rn4, mm8, bosTau2, loxAfr1, echTel1 \\
\hline & & 1: 214745751 - 214745779 & $1124-1152$ & $\begin{array}{l}\text { rn4, mm8, oryCun1, bosTau2, canFam2, loxAfr1, echTel1 } \\
\text { monDom4, galGal2, xenTro1 }\end{array}$ \\
\hline & & $1: 214744387-214744415$ & $2488-2516$ & $\begin{array}{l}\text { rn4, mm8, oryCun1, bosTau2, canFam2, dasNov1, } \\
\text { loxAfr1, echTel1, monDom4, galGal2 }\end{array}$ \\
\hline & & 1: $214745953-214745981$ & $922-950$ & oryCun1, bosTau2, loxAfr1 \\
\hline \multirow{4}{*}{2} & \multirow{4}{*}{ 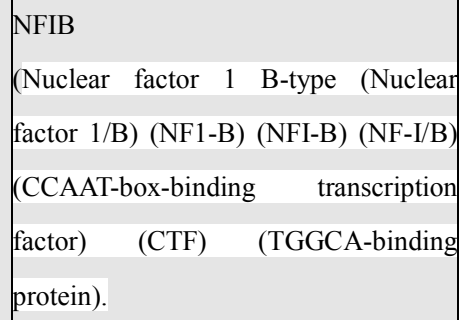 } & 9: $14073314-14073342$ & $4965-4993$ & bosTau2, canFam2, dasNov1, echTel1, galGal2, xenTro1 \\
\hline & & 9: $14077424-14077452$ & $855-883$ & $\begin{array}{l}\text { rn4, mm8, oryCun1, bosTau2, canFam2, dasNov1, } \\
\text { loxAfr1, monDom4, galGal2 }\end{array}$ \\
\hline & & 9: $14073041-14073069$ & $5238-5266$ & $\begin{array}{l}\text { rn4, mm8, oryCun1, bosTau2, canFam2, echTel1, } \\
\text { monDom4, galGal2, xenTro1 }\end{array}$ \\
\hline & & 9: $14073133-14073161$ & $5146-5174$ & $\mathrm{~mm} 8$, bosTau2, canFam2, monDom4, xenTro1 \\
\hline \multirow{5}{*}{3} & \multirow{5}{*}{$\mid \begin{array}{lll}\text { VCPIP1 } & \\
\text { (Deubiquitinating } & \text { protein VCIP135 } \\
\text { (EC } & 3.4 .22 .-) & \text { (Valosin-containing } \\
\text { protein } & \text { p97/p47 } & \text { complex-interacting } \\
\text { protein } & \text { p135) } & \text { (Valosin-containing }\end{array}$} & 8: $67707963-67707991$ & $1298-1326$ & $\mathrm{rn} 4$ \\
\hline & & 8: $67706404-67706432$ & $2857-2885$ & bosTau2, canFam2 \\
\hline & & 8: $67706812-67706840$ & $2449-2477$ & Not Conserved \\
\hline & & 8: $67706374-67706402$ & $2887-2915$ & Not Conserved \\
\hline & & 8: $67703330-67703358$ & $5931-5959$ & Not Conserved \\
\hline \multirow{3}{*}{4} & \multirow{3}{*}{$\begin{array}{l}\text { SATB1 } \\
\text { (DNA-binding protein SATB1 (Special } \\
\text { AT-rich sequence-binding protein 1). }\end{array}$} & 3: $18364785-18364813$ & $852-880$ & $\begin{array}{l}\text { rn4, mm8, oryCun1, bosTau2, canFam2, dasNov1, } \\
\text { loxAfr1, echTel1, monDom4, galGal2, xenTro1 }\end{array}$ \\
\hline & & $13: 18364748-18364776$ & $889-917$ & $\begin{array}{l}\text { rn4, mm8, oryCun1, bosTau2, canFam2, loxAfr1, echTel1, } \\
\text { monDom4, galGal2, xenTro1, tetNig1 }\end{array}$ \\
\hline & & 3: $18364682-18364710$ & $955-983$ & $\begin{array}{l}\text { rn4, mm8, oryCun1, bosTau2, canFam2, dasNov1, } \\
\text { loxAfr1, echTel1, monDom4, galGal2, xenTro1 }\end{array}$ \\
\hline \multirow{4}{*}{5} & \multirow{4}{*}{$\begin{array}{l}\text { SGK269 } \\
\text { (Tyrosine-protein kinase SgK269 (EC } \\
\text { 2.7.10.2) (Sugen kinase 269). }\end{array}$} & 15: $75192237-75192265$ & $1287-1315$ & rn4, mm8, bosTau2, dasNov1, galGal2 \\
\hline & & 15: $75192695-75192723$ & $829-857$ & mm8, loxAfr1 \\
\hline & & 15: $75189373-75189401$ & $4151-4179$ & Not Conserved \\
\hline & & 15: $75190325-75190353$ & $3199-3227$ & Not Conserved \\
\hline \multirow{3}{*}{6} & \multirow{3}{*}{ 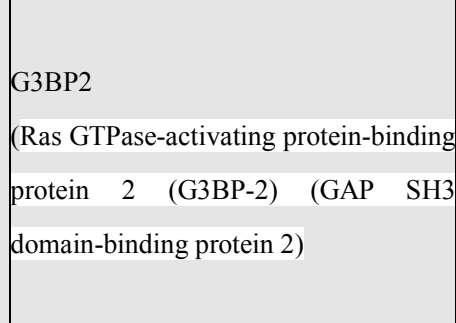 } & 4: $76789250-76789278$ & $359-387$ & $\begin{array}{l}\text { rn4, mm8, oryCun1, bosTau2, canFam2, dasNov1, } \\
\text { loxAfr1, echTel1, monDom4, galGal2, tetNig1 }\end{array}$ \\
\hline & & 4: 76789241 - 76789269 & $368-396$ & $\begin{array}{l}\text { rn4, mm8, bosTau2, canFam2, loxAfr1, echTel1, } \\
\text { monDom4 }\end{array}$ \\
\hline & & 4: $76788319-76788347$ & $1290-1318$ & $\begin{array}{l}\text { rn4, mm8, oryCun1, bosTau2, canFam2, monDom4, } \\
\text { galGal2 }\end{array}$ \\
\hline \multirow{4}{*}{7} & \multirow{4}{*}{$\begin{array}{l}\text { ONECUT2 } \\
\text { (One cut domain family member } 2 \\
\text { (Transcription factor ONECUT-2) } \\
\text { (OC-2). }\end{array}$} & 18: $53302147-53302175$ & $7193-7221$ & rn4, oryCun1, bosTau2, canFam2, echTel1 \\
\hline & & 18: $53304701-53304729$ & 9747 - 9775 & rn4, mm8, bosTau2, dasNov1, loxAfr1 \\
\hline & & 18: $53309471-53309499$ & $14517-14545$ & oryCun1, bosTau2, canFam2, loxAfr1, xenTro1 \\
\hline & & 18: $53307520-53307548$ & $12566-12594$ & Not Conserved \\
\hline \multirow{4}{*}{8} & \multirow{4}{*}{$\begin{array}{l}\text { BCAT1 } \\
\text { (Branched-chain-amino-acid } \\
\text { aminotransferase) }\end{array}$} & 12: $24859551-24859579$ & $2629-2657$ & mm8, oryCun1, bosTau2, canFam2, loxAfr1, echTel1 \\
\hline & & 12: 24859509 - 24859537 & $2671-2699$ & bosTau2, dasNov1, loxAfr1, echTel1 \\
\hline & & 12: $24856005-24856033$ & $6175-6203$ & loxAfr1, galGal2 \\
\hline & & 12: $24861843-24861871$ & $337-365$ & Not Conserved \\
\hline
\end{tabular}




\begin{tabular}{|c|c|c|c|c|}
\hline \multirow{6}{*}{9} & PDS5B & 13: $32248010-32248038$ & $819-847$ & rn4, mm8, oryCun1, bosTau2, canFam2, dasNov1, loxAfr1 \\
\hline & (Androgen-induced & $13: 32249400-32249428$ & $2209-2237$ & $\mathrm{rn} 4, \mathrm{~mm} 8$ \\
\hline & inhibitor (Androgen-induced prostate & $13: 32247595-32247623$ & $404-432$ & rn4, mm8, oryCun1, canFam2, dasNov1 \\
\hline & proliferative shutoff-associated protein & & & \\
\hline & AS3) (Sister chromatid cohesion & $13: 32248890-32248918$ & $1699-1727$ & Not Conserved \\
\hline & protein PDS5 homolog B). & & & \\
\hline \multirow{4}{*}{$1 \mathrm{c}$} & \multirow{4}{*}{$\begin{array}{l}\text { TNRC6B } \\
\text { (Trinucleotide }\end{array}$} & 22: $39053089-39053117$ & $3897-3925$ & bosTau2, canFam2, dasNov1, loxAfr1 \\
\hline & & 22: $39051494-39051522$ & $2302-2330$ & Not Conserved \\
\hline & & 22: $39057884-39057912$ & $8692-872$ & Not Conserved \\
\hline & & 22: 39058466 - 39058494 & $9274-9302$ & Not Conserved \\
\hline \multirow{5}{*}{11} & GRK7 & 3: $143018910-143018938$ & $327-355$ & oryCun1, canFam2, dasNov1, echTel1, monDom4 \\
\hline & (G protein-coupled receptor kinase 7 & 73: $143019117-143019145$ & $534-562$ & Not Conserved \\
\hline & 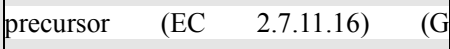 & & & \\
\hline & protein-coupled receptor kinase & 3: $143019734-143019762$ & $1151-1179$ & Not Conserved \\
\hline & GRK7). & & & \\
\hline \multirow{4}{*}{12} & EEA1 & 12: 91690948 - 91690976 & $2941-2969$ & canFam2, dasNov1, loxAfr1, echTel1 \\
\hline & (Early endosome antigen & 12: $91690576-91690604$ & $3313-3341$ & oryCun1 \\
\hline & (Endosome-associated protein p162) & 12: $91693577-91693605$ & $312-340$ & Not Conserved \\
\hline & $\begin{array}{l}\text { (Zinc finger FYVE domain-containing } \\
\text { protein 2). }\end{array}$ & 12: $91690736-91690764$ & $3153-3181$ & Not Conserved \\
\hline \multirow{5}{*}{13} & SOCS5 & D. $46842834-46842862$ & b049-2077 & rn4, mm8, bosTau2, canFam2, dasNov1, loxAfr1, echTel1, \\
\hline & (Suppressor of cytokine signaling 5 & & & monDom4 \\
\hline & (SOCS-5) (Cytokine-inducible SH2- & $-2: 46841257-46841285$ & $472-500$ & mm8, oryCun1, canFam2, dasNov1, loxAfr1 \\
\hline & containing & 2: $46841767-46841795$ & $982-1010$ & echTel1 \\
\hline & (Cytokine-inducible SH2 protein 6) & 2: $46843207-46843235$ & $2422-2450$ & echTel1 \\
\hline & & miRNA5_GA_CONTIG1 ( & CAUGCCUC & UCCGAGC) \\
\hline \multirow{7}{*}{1} & \multirow{7}{*}{$\begin{array}{l}\text { ZNF677 } \\
\text { (Zinc finger protein } 677 .)\end{array}$} & 19: $58431395-58431423$ & $615-643$ & Not Conserved \\
\hline & & 19: $58431140-58431168$ & $870-898$ & Not Conserved \\
\hline & & 19: $58430990-58431018$ & $1020-1048$ & Not Conserved \\
\hline & & 19: $58429002-58429030$ & $2602-2630$ & Not Conserved \\
\hline & & 19: $58427732-58427760$ & $3872-3900$ & Not Conserved \\
\hline & & 19: $58426257-58426285$ & $5347-5375$ & Not Conserved \\
\hline & & $\begin{array}{l}19: 58423998 ; 58425445 \\
58424011 ; 58425460\end{array}$ & $6172-6200$ & Not Conserved \\
\hline \multirow{5}{*}{2} & & 17: $26884596-26884624$ & $1725-1753$ & dasNov1 \\
\hline & RAB11FIP4 & 17: $26885526-26885554$ & $2655-2683$ & Not Conserved \\
\hline & (Rab11 family-interacting protein 4 & 17: $26885656-26885684$ & $2785-2813$ & Not Conserved \\
\hline & (Rab11-FIP4). & 17: $26885770-26885798$ & $2899-2927$ & Not Conserved \\
\hline & & 17: $26886259-26886287$ & $3388-3416$ & Not Conserved \\
\hline \multirow{2}{*}{3} & SPN & 16: 29585767 - 29585795 & $2013-2041$ & Not Conserved \\
\hline & (Leukosialin precursor (Leukocyte & $16: 29586434-29586462$ & $2680-2708$ & Not Conserved \\
\hline
\end{tabular}




\begin{tabular}{|c|c|c|c|c|}
\hline & sialoglycoprotein) & 16: $29587132-29587160$ & $3378-3406$ & Not Conserved \\
\hline & (Galactoglycoprotein) & 16: $29588144-29588172$ & $4390-4418$ & Not Conserved \\
\hline & \multirow[t]{2}{*}{ (CD43 antigen). } & 16: 29589017 - 29589045 & $5263-5291$ & Not Conserved \\
\hline & & 16: 29589155 - 29589183 & $5401-5429$ & Not Conserved \\
\hline \multirow{4}{*}{4} & \multirow{4}{*}{$\begin{array}{l}\text { ZNF529 } \\
\text { (Zinc finger protein 529. ) }\end{array}$} & 19: $41728138-41728166$ & $1441-1469$ & Not Conserved \\
\hline & & 19: $41728004-41728032$ & $1575-1603$ & Not Conserved \\
\hline & & 19: $41727831-41727859$ & $1748-1776$ & Not Conserved \\
\hline & & 19: 41727702 - 41727730 & $1877-1905$ & Not Conserved \\
\hline \multirow{6}{*}{5} & \multirow{6}{*}{$\begin{array}{l}\mathrm{ZC} 3 \mathrm{H} 6 \\
\text { (Zinc finger } \mathrm{CCCH} \text { domain-containing } \\
\text { protein } 6 .\end{array}$} & 2: $112810108-112810136$ & $3571-3599$ & dasNov1 \\
\hline & & 2: $112811625-112811653$ & $5088-5116$ & Not Conserved \\
\hline & & 2: $112811925-112811953$ & $5388-5416$ & Not Conserved \\
\hline & & 2: $112812117-112812145$ & $5580-5608$ & Not Conserved \\
\hline & & 2: $112812253-112812281$ & $5716-5744$ & Not Conserved \\
\hline & & 2: $112813197-112813225$ & $6660-6688$ & Not Conserved \\
\hline \multirow{6}{*}{6} & \multirow{6}{*}{$\begin{array}{l}\text { ZNF587 } \\
\text { (Zinc finger protein 587.) }\end{array}$} & 19: $63063703-63063731$ & $382-410$ & Not Conserved \\
\hline & & 19: $63063768-63063796$ & $447-475$ & Not Conserved \\
\hline & & 19: $63064631-63064659$ & $1310-1338$ & Not Conserved \\
\hline & & 19: $63065070-63065098$ & $1749-1777$ & Not Conserved \\
\hline & & 19: $63066551-63066579$ & $3230-3258$ & Not Conserved \\
\hline & & 19: $63067634-63067662$ & $4313-4341$ & Not Conserved \\
\hline \multirow{4}{*}{7} & \multirow{4}{*}{$\begin{array}{l}\text { ANGEL2 } \\
\text { (Protein angel homolog 2.) }\end{array}$} & $1: 211234115-211234143$ & $862-890$ & Not Conserved \\
\hline & & 1: $211233364-211233392$ & $1613-1641$ & oryCun1, canFam2 \\
\hline & & 1: $211233143-211233171$ & $1834-1862$ & Not Conserved \\
\hline & & 1: $211233006-211233034$ & $1971-1999$ & Not Conserved \\
\hline \multirow{5}{*}{8} & \multirow{5}{*}{$\begin{array}{lr}\text { NF2 } & \\
\text { (Merlin } & \text { (Moesin-ezrin-radixin-lik } \\
\text { protein) } & \text { (Neurofibromin-2 } \\
\text { (Schwannomin) } & \text { (Schwannomerlin) }\end{array}$} & 22: $28421224-28421252$ & $781-809$ & Not Conserved \\
\hline & & 22: 28422186 - 28422214 & $1743-1771$ & Not Conserved \\
\hline & & $22: 28422286-28422314$ & $1843-1871$ & Not Conserved \\
\hline & & 22: $28422929-28422957$ & $2486-2514$ & Not Conserved \\
\hline & & 22: $28423813-28423841$ & $3370-3398$ & Not Conserved \\
\hline \multirow{5}{*}{9} & \multirow{5}{*}{$\begin{array}{l}\text { KIAA1217 } \\
\text { (Sickle tail protein homolog) }\end{array}$} & 10: $24874195-24874223$ & $141-169$ & $\begin{array}{l}\text { rn4, mm8, bosTau2, canFam2, dasNov1, echTel1, } \\
\text { monDom4, galGal2, xenTro1 }\end{array}$ \\
\hline & & 10: $24876216-24876244$ & $2162-2190$ & $\begin{array}{l}\text { rn4, mm8, bosTau2, canFam2, loxAfr1, echTel1, } \\
\text { monDom4, galGal2, tetNig1 }\end{array}$ \\
\hline & & 10: $24876389-24876417$ & $2335-2363$ & $\begin{array}{l}\text { rn4, mm8, bosTau2, canFam2, loxAfr1, echTel1, } \\
\text { monDom4, galGal2 }\end{array}$ \\
\hline & & 10: $24874144-24874172$ & $90-118$ & rn4, mm8, bosTau2, canFam2, echTel1, monDom4, galGal2 \\
\hline & & 10: $24875849-24875877$ & $1795-1823$ & Not Conserved \\
\hline \multirow{4}{*}{10} & \multirow{4}{*}{$\begin{array}{l}\text { SLC7A2 } \\
\text { (Low-affinity cationic amino acid } \\
\text { transporter 2 (CAT-2) (CAT2) }\end{array}$} & 8: 17468612 - 17468640 & $1682-1710$ & Not Conserved \\
\hline & & 8: $17468959-17468987$ & $2029-2057$ & Not Conserved \\
\hline & & 8: $17470445-17470473$ & $3515-3543$ & Not Conserved \\
\hline & & 8: $17470592-17470620$ & $3662-3690$ & Not Conserved \\
\hline
\end{tabular}




\begin{tabular}{|c|c|c|c|c|}
\hline \multirow{5}{*}{11} & \multirow{5}{*}{$\begin{array}{l}\text { ZFP14 } \\
\text { (Zinc finger protein } 14 \text { homolog } \\
\text { (Zfp-14) (Zinc finger protein 531). }\end{array}$} & 19: $41522534-41522562$ & $403-431$ & Not Conserved \\
\hline & & 19: $41522286-41522314$ & $651-67$ & Not Conserved \\
\hline & & 19: $41521162-41521190$ & $1775-1803$ & Not Conserved \\
\hline & & 19: $41520886-41520914$ & $2051-2079$ & Not Conserved \\
\hline & & 19: $41519135-41519163$ & $3802-3830$ & Not Conserved \\
\hline \multirow{5}{*}{12} & \multirow{5}{*}{$\begin{array}{l}\text { LONRF2 } \\
\text { (LON peptidase N-terminal domain } \\
\text { and RING finger protein } 2 \text { (RING } \\
\text { finger protein 192) (Neuroblastoma } \\
\text { apoptosis-related protease). }\end{array}$} & 2: $100267134-100267162$ & $29-57$ & Not Conserved \\
\hline & & 2: $100264591-100264619$ & $2572-2600$ & Not Conserved \\
\hline & & $2: 100263404-10026343$ & $3759-3787$ & Not Conserved \\
\hline & & $2: 100260564-100260592$ & $6599-6627$ & Not Conserved \\
\hline & & 2: $100257882-100257910$ & $9281-9309$ & Not Conserved \\
\hline \multirow{4}{*}{13} & \multirow{4}{*}{$\begin{array}{l}\text { C20orf142 } \\
\text { (Uncharacterized protein C20orf142 } \\
\text { precursor.) }\end{array}$} & 20: $42368233-42368261$ & $417-445$ & Not Conserved \\
\hline & & 20: $42367745-42367773$ & $905-933$ & Not Conserved \\
\hline & & $20: 42367611-42367639$ & $1039-1067$ & Not Conserved \\
\hline & & 20: $42365958-42365986$ & $2692-2720$ & Not Conserved \\
\hline \multirow{5}{*}{14} & \multirow{5}{*}{$\begin{array}{l}\text { ZNF284 } \\
\text { (Zinc finger protein 284.) }\end{array}$} & 19: $49284018-49284046$ & $764-792$ & Not Conserved \\
\hline & & 19: $49284161-49284189$ & $907-935$ & Not Conserved \\
\hline & & 19: $49284840-49284868$ & $1586-1614$ & Not Conserved \\
\hline & & 19: $49284933-49284961$ & $1679-1707$ & Not Conserved \\
\hline & & 19: $49285240-49285268$ & $1986-2014$ & Not Conserved \\
\hline \multirow{5}{*}{15} & \multirow{5}{*}{$\begin{array}{l}\text { MDN1 } \\
\text { (Midasin (MIDAS-containing protein). }\end{array}$} & 6: 90459275 - 90459303 & $6443-6471$ & $\begin{array}{l}\text { rn4, mm8, oryCun1, bosTau2, dasNov1, loxAfr1, echTel1, } \\
\text { monDom4, galGal2, xenTro1, tetNig1 }\end{array}$ \\
\hline & & 6: 90510050 - 90510078 & $564-592$ & $\begin{array}{l}\text { rn4, mm8, oryCun1, bosTau2, canFam2, dasNov1, echTel1, } \\
\text { monDom4, xenTro1 }\end{array}$ \\
\hline & & 6: 90499093 - 90499121 & $1123-1151$ & $\begin{array}{l}\text { rn4, mm8, oryCun1, canFam2, dasNov1, loxAfr1, echTel1, } \\
\text { monDom4 }\end{array}$ \\
\hline & & 6: $90511937-90511965$ & $317-345$ & bosTau2, loxAfr1, echTel1 \\
\hline & & 6: $90442601-90442629$ & $8820-8848$ & canFam2 \\
\hline \multirow{6}{*}{16} & \multirow{6}{*}{$\begin{array}{l}\text { ZFTB8 } \\
\text { (zinc finger and BTB }\end{array}$} & 1: $32838716-32838744$ & $108-136$ & $\begin{array}{l}\text { rn4, mm8, oryCun1, bosTau2, canFam2, dasNov1, loxAfr1, } \\
\text { echTel1, monDom4 }\end{array}$ \\
\hline & & 1: $32840994-32841022$ & $2386-2414$ & $\begin{array}{l}\text { rn4, mm8, oryCun1, bosTau2, canFam2, dasNov1, loxAfr1, } \\
\text { echTel1, monDom4, galGal2, xenTro1 }\end{array}$ \\
\hline & & $1: 32841438-32841466$ & $2830-2858$ & Not Conserved \\
\hline & & 1: $32841571-32841599$ & $2963-2991$ & Not Conserved \\
\hline & & 1: $32843593-32843621$ & $4985-5013$ & Not Conserved \\
\hline & & 1: $32843729-32843757$ & $5121-5149$ & Not Conserved \\
\hline \multirow{4}{*}{17} & \multirow{4}{*}{$\begin{array}{l}\text { SGCB } \\
\text { (Beta-sarcoglycan } \\
\text { dystrophin-associ } \\
\text { (43DAG) (A3b). }\end{array}$} & 4: $52584823-52584851$ & $28-56$ & $\begin{array}{l}\text { oryCun1, bosTau2, dasNov1, loxAfr1, echTel1, monDom4, } \\
\text { galGal2 }\end{array}$ \\
\hline & & 4: $52584017-52584045$ & $834-862$ & Not Conserved \\
\hline & & 4: $52583591-52583619$ & $1260-1288$ & Not Conserved \\
\hline & & 4: $52582449-52582477$ & $2402-2430$ & Not Conserved \\
\hline \multirow{2}{*}{18} & CHST6 & 16: $74068297-74068325$ & $1714-1742$ & Not Conserved \\
\hline & (Carbohydrate sulfotransferase 6 (EC & $16: 74067685-74067713$ & $2326-2354$ & Not Conserved \\
\hline
\end{tabular}




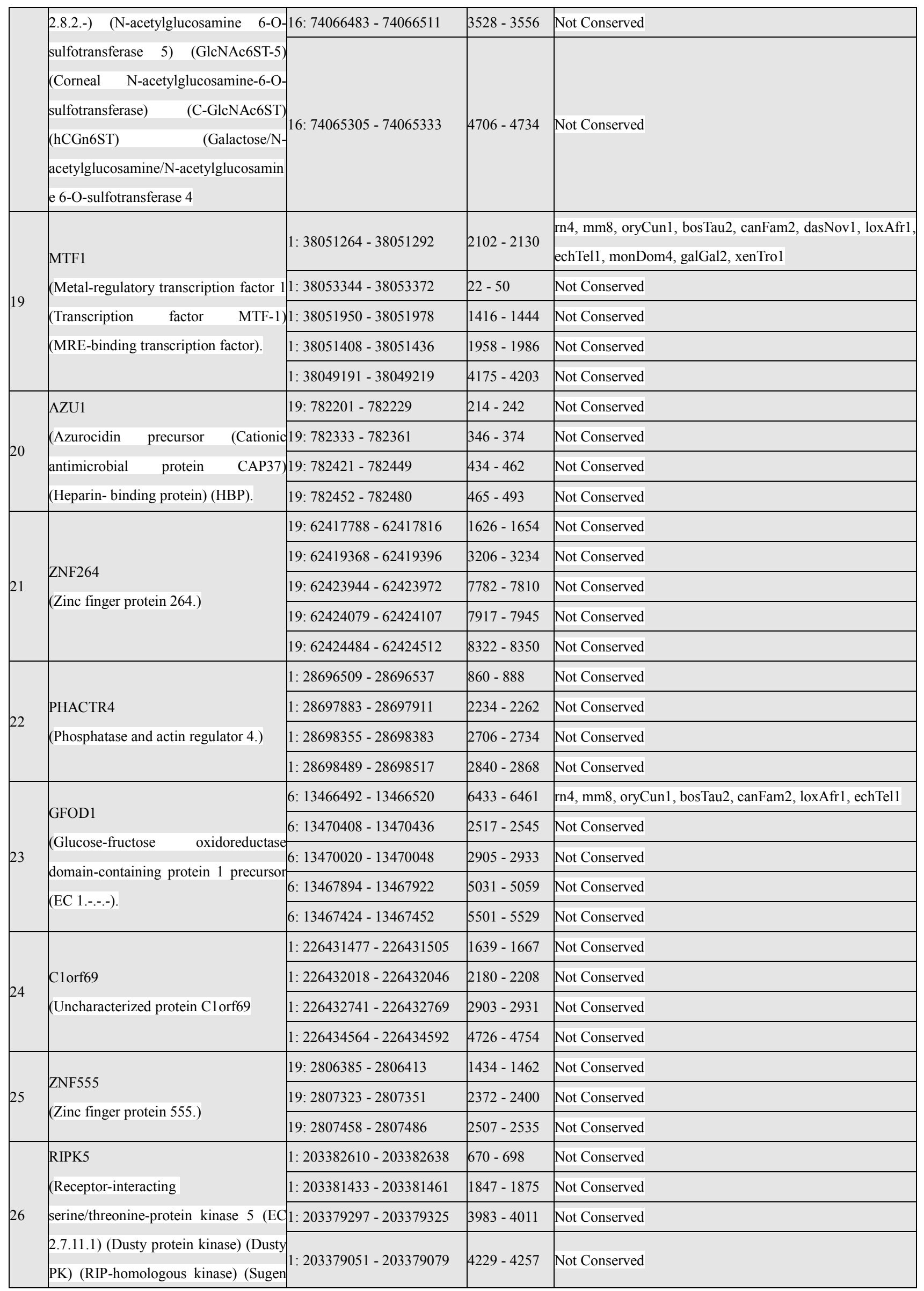




\begin{tabular}{|c|c|c|c|c|}
\hline & kinase 496). & & & \\
\hline \multirow{4}{*}{2} & \multirow{4}{*}{$\begin{array}{l}\text { FLJ45803 } \\
\text { (CDNA FLJ45803 fis) }\end{array}$} & 11: $110671723-110671751$ & $287-315$ & Not Conserved \\
\hline & & 11: $110671509-110671537$ & $501-529$ & Not Conserved \\
\hline & & 11: $110671376-110671404$ & $634-662$ & Not Conserved \\
\hline & & 11: $110670401-110670429$ & $1609-163$ & Not Conserved \\
\hline \multirow{3}{*}{28} & TNRC6B & 22: 39049792 - 39049820 & $600-628$ & $\begin{array}{l}\text { rn4, mm8, oryCun1, canFam2, dasNov1, loxAfr1, echTel1, } \\
\text { monDom4 }\end{array}$ \\
\hline & (Trinucleotide repeat-containing 6B & 22: $39051365-39051393$ & $2173-2201$ & Not Conserved \\
\hline & Proming) & 22: $39058550-39058578$ & $9358-9386$ & mm8, oryCun1, loxAfr1 \\
\hline \multirow{4}{*}{29} & \multirow{4}{*}{$\begin{array}{l}\text { C1orf216 } \\
\text { (CDNA FLJ39147 fis) }\end{array}$} & 1: $35953768-35953796$ & $23-51$ & oryCun1, canFam2 \\
\hline & & 1: 35953714 - 35953742 & $77-105$ & Not Conserved \\
\hline & & 1: $35953672-35953700$ & $119-147$ & Not Conserved \\
\hline & & 1: $35953359-35953387$ & $432-460$ & dasNov1 \\
\hline \multirow{4}{*}{30} & \multirow{4}{*}{ (CDNA FLJ45156 fis) } & 1: $159484654-159484682$ & $606-634$ & Not Conserved \\
\hline & & 1: $159485346-159485374$ & $1298-1326$ & Not Conserved \\
\hline & & 1: $159485948-159485976$ & $1900-1928$ & Not Conserved \\
\hline & & 1: $159486082-159486110$ & $2034-2062$ & Not Conserved \\
\hline \multirow{4}{*}{31} & \multirow{4}{*}{$\begin{array}{l}\text { GJA7 } \\
\text { (Gap junction alp } \\
\text { (Connexin-45) (Cx45) }\end{array}$} & 17: 40235766 - 40235794 & $1726-1754$ & Not Conserved \\
\hline & & 17: $40233290-40233318$ & $4202-4230$ & Not Conserved \\
\hline & & 17: $40232208-40232236$ & $5284-5312$ & Not Conserved \\
\hline & & 17: $40231872-40231900$ & $5620-5648$ & Not Conserved \\
\hline \multirow[b]{3}{*}{32} & RSL1D1 & 16: $11838477-11838505$ & $639-667$ & Not Conserved \\
\hline & (Ribosomal L1 domain-containing & 16: $11836693-11836721$ & $2423-2451$ & Not Conserved \\
\hline & $\begin{array}{l}\text { protein } 1 \text { (Cellular senescence- } \\
\text { inhibited gene protein) (Protein PBK1) } \\
\text { (CATX-11). }\end{array}$ & $16: 11836336-11836364$ & $2780-2808$ & Not Conserved \\
\hline \multirow{3}{*}{33} & ENDOGL1 & 3: 38540876 - 38540904 & $18-46$ & Not Conserved \\
\hline & (Endonuclease G-like 1 (EC 3.1.30.-) & $3: 38541861-38541889$ & $1003-1031$ & Not Conserved \\
\hline & (Endo G-like). & 3: $38542139-38542167$ & $1281-1309$ & Not Conserved \\
\hline \multirow{3}{*}{34} & NT5DC3 & 12: $102695687-102695715$ & $21-49$ & Not Conserved \\
\hline & (5'-nucleotidase domain containing 3 & 12: $102694332-102694360$ & $1376-1404$ & Not Conserved \\
\hline & isoform 1 ) & 12: $102692324-102692352$ & $3384-3412$ & Not Conserved \\
\hline \multirow{4}{*}{35} & GMEMB1 & 1: $28914174-28914202$ & $301-329$ & Not Conserved \\
\hline & (Glucocorticoid & $1: 28914360-28914388$ & $487-515$ & oryCun1 \\
\hline & element-binding protein 1 (GMEB-1) & $1: 28916544-28916572$ & $2671-2699$ & Not Conserved \\
\hline & $\begin{array}{l}\text { (Parvovirus initiation factor p96) (PIF } \\
\text { p96) (DNA-binding protein p96PIF) }\end{array}$ & $1: 28917401-28917429$ & $3528-3556$ & Not Conserved \\
\hline \multicolumn{5}{|c|}{ >miRNA6_GA_CONTIG1 (ACUCAAGACUCUUUUGUUCU) } \\
\hline \multirow{4}{*}{1} & NTRK3 & 15: $86216493-86216521$ & $4648-4676$ & bosTau2, canFam2 \\
\hline & (NT-3 growth factor receptor precursor & 15: $86212170-86212198$ & $8971-8999$ & bosTau2 \\
\hline & (EC 2.7.10.1) (Neurotrophic tyrosine & $15: 86211855-86211883$ & $9286-9314$ & Not Conserved \\
\hline & kinase receptor type 3) (TrkC tyrosine & $15: 86211054-86211082$ & $10087-10115$ & dasNov1, loxAfr1 \\
\hline
\end{tabular}




\begin{tabular}{|c|c|c|c|c|}
\hline & kinase) (GP145-TrkC) (Trk-C). & 15: $86206441-86206469$ & $14700-14728$ & Not Conserved \\
\hline \multirow{4}{*}{2} & \multirow{4}{*}{$\begin{array}{l}\text { NUAK family SNF1-like kinase } 1 \text { (EC } \\
\text { 2.7.11.1) (AMPK-related protein }\end{array}$} & 12: $104981720-104981748$ & $2961-2989$ & rn4, mm8, oryCun1, bosTau2, canFam2, dasNov1, loxAfr1 \\
\hline & & 12: $104983004-104983032$ & $1677-1705$ & bosTau2 \\
\hline & & 12: $104984225-104984253$ & $456-484$ & Not Conserved \\
\hline & & 12: $104983836-104983864$ & $845-873$ & Not Conserved \\
\hline 3 & \multirow{4}{*}{$\begin{array}{l}\text { ZNF578 } \\
\text { (zinc finger protein } 765 \text { ) }\end{array}$} & 19: $58604982-58605010$ & $789-817$ & Not Conserved \\
\hline & & 19: $58604353-58604381$ & $160-188$ & Not Conserved \\
\hline & & 19: $58606114-58606142$ & $1921-1949$ & Not Conserved \\
\hline & & 19: $58606244-58606272$ & $2051-2079$ & Not Conserved \\
\hline \multirow{7}{*}{4} & PAG1 & 8: $82048478-82048506$ & $2827-2855$ & loxAfr1 \\
\hline & (Phosphoprotein associated with & 8: $82047549-82047577$ & $3756-3784$ & oryCun1, loxAfr1 \\
\hline & glycosphingolipid-enriched & & & \\
\hline & microdomains 1 (Transmembrane & 8: $82043898-82043926$ & $7407-7435$ & Not Conserved \\
\hline & adapter protein PAG) (Csk-binding & & & \\
\hline & (Transmembrane & 8: $82043625-82043653$ & $7680-7708$ & Not Conserved \\
\hline & phosphoprotein Cbp). & & & \\
\hline \multirow{7}{*}{5} & PRDM2 & 1: $14022570-14022598$ & $351-379$ & Not Conserved \\
\hline & (PR domain zinc finger protein 2 (PR & $1: 14022812-14022840$ & $593-621$ & bosTau2 \\
\hline & domain-containing protein 2) & 1: $14022855-14022883$ & $636-664$ & loxAfr1 \\
\hline & $\begin{array}{l}\text { (Retinoblastoma protein-interacting } \\
\text { zinc finger protein) (Zinc finger protein }\end{array}$ & \multirow{4}{*}{ 1: $14023828-14023856$} & \multirow{4}{*}{$1609-1637$} & \multirow{4}{*}{ bosTau2, canFam2, loxAfr1 } \\
\hline & RIZ) (MTE-binding & & & \\
\hline & (MTB-ZF) (GATA-3-binding protein & & & \\
\hline & G3B). & & & \\
\hline \multirow{4}{*}{6} & \multirow{4}{*}{$\begin{array}{l}\text { SETD7 } \\
\text { (Histone-lysine N-methyltransferase) }\end{array}$} & 4: $140651343-140651371$ & $895-923$ & Not Conserved \\
\hline & & 4: $140649691-140649719$ & $2547-2575$ & Not Conserved \\
\hline & & 4: $140648972-140649000$ & $3266-3294$ & Not Conserved \\
\hline & & 4: $140647315-140647343$ & $4923-4951$ & $\mathrm{~mm} 8$, bosTau2, dasNov1 \\
\hline
\end{tabular}

Table 2 explains: name of genes and position on chromosomes with UTRs and gene target information given by conserved information and this prediction analysis mainly completed by DIANA tar with KEGG analysis for metabolomic activity .yellow color highlighter shows the miRNA with nucleotide sequences.

\section{Discussion and Conclusion}

Human 3UTRs were found via the DIANA tar total 6 miRNA were found to hybridizes with human 3UTR.with the help of KEGG pathway in a genes for various metabolic pathways in human were tracked .this might silence the gene expression of disease like cancer, blood borne disease, , diuretic, anthelmintic activity, cardio protective, anti diabetic, immunomodulatory, and many more disease by gene silencing the query whether the miRNAs are blocking or repressing any of the human genes was solved by hybridizing putative miRNAs with 3UTR of human through DIANA tar web server. Present studies shows that Gmelina arborea is a very important medicinal plant for human against various diseases. The miRNA predicted from it were found to target various genes on metabolic pathway the study concludes that this first reporting of the scientific exploration of multiple action potential of Gmelina arborea miRNA with special reference in cancer and blood brone disease models is not only interesting but very encouraging too for medicinal chemists as well as cancer patients globally. These predicted miRNA may serve as potential resource for initiating experimental validation which may provide valuable information for functional analysis of miRNA regulated pathway.

\section{Acknowledgements}

It is my proud privilege to express deepest sense of reverence and heart full thanks DBT, for providing me scientific computational platform 


\section{References}

[1] VL Saxena and A Dwivedi ,insilico identification of miRNA and their target prediction from Japanese encephalitis. Journal of Bioinformatics and Sequence Analysis, 2013, Vol. 5(2), pp. 25-33.

[2] Bartel DP , "MicroRNAs: target recognition and regulatory functions, 2009, Cell 136(2): 215-33.

[3] Lelandais-Brière C, Sorin C, Declerck M, Benslimane A, Crespi M, Hartmann C ,Small RNA diversity in plants and its impact in development ,2010,Current Genomics 11 (1): $14-23$.

[4] A.S.R. Anjaneyulu, A.Madhusudhana rao, V.Kameswara Rao and L.Ramachandra, Novel hydroxy lignans from the heartwood of gmelina arborea Row, Tetrahedron, 1977, Volume 33, Issue 1, Pages 133-143,

[5] Rashmi Rameshwari1, Divya Singhal, Rachit Narang,Apurvi Maheshwari, and T. V. Prasad, IN SILICO PREDICTION OF MIRNA IN Curcuma longa AND THEIR ROLE IN HUMAN METABOLOMICS. International Journal of Advanced Biotechnology and Research,2013 Vol 4, Issue 2, pp 253-259.

[6] Falgueras J, SeqTrim, a high-throughput pipeline for pre-processing any type of sequence read., 2010,BMC Bioinformatics, 11:38

[7] Geo Pertea, TIGR Gene Indicesclustering tools (TGICL), a software system forfast clustering of large EST datasets. Bioinformatics. 2002, Vol. 19, issue 5.

[8] Adams MD, Kelley JM, Gocayne JD, Complementary DNA sequencing: expressed sequence tags and human genome project ,1991,Science 252 (2013): 1651-6. 